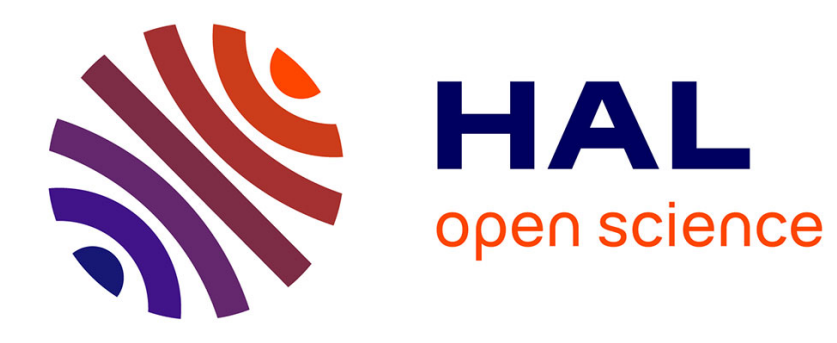

\title{
Hans-Jörg Rheinberger, Introduction à la philosophie des sciences
}

Maël Dieudonné

\section{To cite this version:}

Maël Dieudonné. Hans-Jörg Rheinberger, Introduction à la philosophie des sciences. 2014, http://lectures.revues.org/14686. halshs-00995116

\section{HAL Id: halshs-00995116 https://shs.hal.science/halshs-00995116}

Submitted on 22 May 2014

HAL is a multi-disciplinary open access archive for the deposit and dissemination of scientific research documents, whether they are published or not. The documents may come from teaching and research institutions in France or abroad, or from public or private research centers.
L'archive ouverte pluridisciplinaire HAL, est destinée au dépôt et à la diffusion de documents scientifiques de niveau recherche, publiés ou non, émanant des établissements d'enseignement et de recherche français ou étrangers, des laboratoires publics ou privés. 


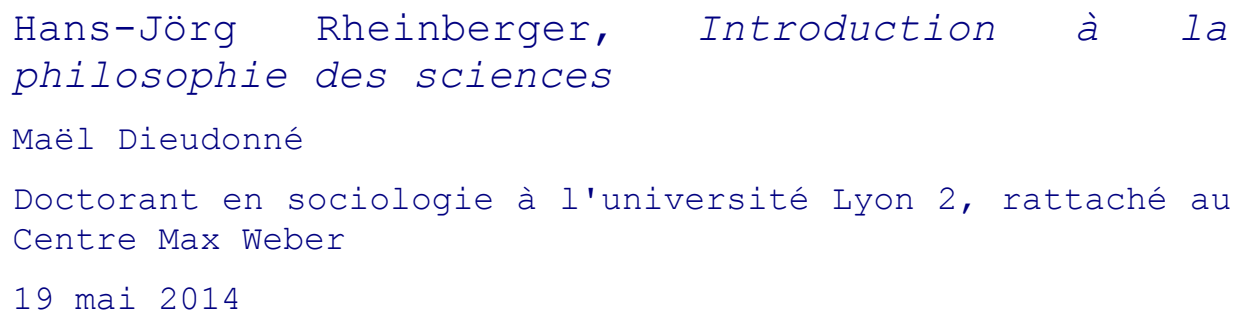

Le champ de l'étude des sciences a connu des développements si nombreux et si variés au cours des dernières décennies que la publication d'un ouvrage permettant de s'y repérer est toujours bienvenue. Une Introduction à la philosophie des sciences, rédigée par l'historien et biologiste allemand Hans-Jörg Rheinberg et traduite de l'allemand par Nathalie Jas, vient ainsi enrichir la collection Repères. Quelle intelligibilité propose-t-elle du versant philosophique de ce champ ? La perspective adoptée est historique, avec un propos organisé chronologiquement et décomposé en six chapitres.

Le premier chapitre est consacré à l'extension de la philosophie mécaniste, à la fin du $\mathrm{XIX}^{\mathrm{e}}$ siècle. Celle-ci exprimait la conviction que les sciences exactes convergeraient tôt ou tard vers le modèle de la physique, à mesure qu'elles parviendraient à décomposer les phénomènes naturels selon leurs plus petites parties, et à les réduire aux forces s'exerçant entre elles (comme le soutenait par exemple l'électrophysiologiste allemand Emil Du Bois-Raymond ${ }^{1}$ ). Mais l'incapacité de la philosophie mécaniste à rendre compte de ses propres concepts (matière, force et mouvement) suscita l'apparition d'une sorte d'agnosticisme épistémologique : elle se trouva conçue comme une fiction justifiée par son utilité, c'est-à-dire comme une convention. De ce mouvement annonçant la possibilité d'une relativisation des exigences du savoir, l'auteur trouve la trace dans les écrits d'Ernst Mach, d'Émile Boutroux, de Whilhelm Dilthey, d'Henri Poincaré et d'Otto Neurath.

Le second chapitre introduit les analyses de Gaston Bachelard et de Ludwig Fleck, qui ont chacune constitué une tentative de prise en compte des deux ruptures consommées dans l'entre-deux-guerres. La première résulte de la Première Guerre mondiale, qui a ruiné la conviction que la science et la technique constituent nécessairement des facteurs de progrès, et attiré l'attention sur les conditions matérielles de production des savoirs. La seconde découle de l'évolution des théories scientifiques elles-mêmes : le développement de la théorie de la relativité, puis celui de la physique quantique, ont imposé l'idée que la connaissance ne s'accumule pas toujours de manière linéaire, et qu'à chaque moment de l'histoire des sciences des alternatives théoriques sont possibles.

Le troisième chapitre présente d'autres analyses développées à la même période, dont les auteurs « ont tous en commun d'avoir contribué à aiguiser la nécessité d'une théorie de la connaissance qui intègre une intelligence de son développement» (p.47) - sans pourtant partager d'autres particularités évidentes. Karl Popper a ainsi souligné l'importance du processus de falsification dans la méthode scientifique;

\footnotetext{
${ }^{1}$ Auquel un ouvrage vient par ailleurs d'être consacré : http://lectures.revues.org/14454
} 
Ernst Cassirer l'a plutôt décrite comme un ensemble de processus d'objectivation ; Edmund Husserl s'est intéressé au rôle de l'écriture dans la sédimentation des connaissances scientifiques, et Martin Heidegger à celle de la technique.

Le quatrième chapitre décrit une nouvelle rupture, opérée après 1945, suite à laquelle la réflexion épistémologique a cessé de se focaliser sur la résolution des difficultés philosophiques soulevées par les évolutions contemporaines des sciences, pour s'emparer d'un questionnement spécifique. Cette évolution a découlé de la subordination nouvelle de la philosophie à l'histoire - qui inversement s'est vu reconnaître un rôle véritablement analytique, au lieu d'une simple fonction d'illustration par la compilation d'anecdotes sur les grandes découvertes. Les plus célèbres artisans de cette rupture furent Thomas Kuhn, Alexandre Koyré, Stephen Toulmin et Paul Feyerabend.

Le cinquième chapitre est consacré au courant post-structuraliste français des années 1960, dont les membres ont selon l'auteur exploré certaines questions fondamentales de la philosophie des sciences. Ainsi Georges Canguilhem a-t-il proposé de concevoir l'histoire des sciences comme une histoire du déplacement de problèmes situés dans des contextes précis, avant que Michel Foucault ne radicalise ce principe en inventant une archéologie du savoir scrupuleusement attentive aux ruptures et aux discontinuités, tandis que Louis Althusser tentait de définir la connaissance comme un processus de production ne pouvant avoir de fin, et que Jacques Derrida soulignait à son tour l'importance de l'écriture dans le travail scientifique.

Le sixième chapitre s'intéresse à une ultime rupture, accomplie dans les années 1980 vis-à-vis de la perspective commune aux auteurs précédents. Elle est également passé par une innovation méthodologique: la réhabilitation de l'anthropologie, et le recentrement de l'attention vers les pratiques les plus concrètes de production des connaissances scientifiques. Ses deux acteurs marquants sont Ian Hacking et Bruno Latour.

Contrairement à ce que laisse penser son titre français, cet ouvrage a été conçu par son auteur comme un essai plutôt que comme un manuel. Le propos étant moins organisé pour présenter intelligiblement une tradition de recherche que pour défendre une thèse, les travaux considérés sont abordés relativement à cette thèse plutôt que selon leurs caractéristiques les plus distinctives. La possession de quelques repères en épistémologie et en histoire des sciences s'avère alors utile pour se repérer dans l'argumentation, ce qui est quelque peu contradictoire pour une introduction. Sur le fond, la thèse d'Hans-Jörg Rheinberger est celle d'une historicisation de l'épistémologie, accomplie brutalement dans la seconde moitié du $\mathrm{XX}^{\mathrm{e}}$ siècle. Sa description est suffisamment minutieuse pour être convaincante. Il est cependant dommage que le nombre d'éléments avancés pour l'expliquer, ainsi que les autres évolutions évoquées, aille décroissant au fil de l'ouvrage (l'introduction générale et celles des deux premiers chapitres sont très éclairantes, mais les suivantes et la conclusion sont plutôt indigentes). Quelques réserves peuvent également être émises concernant le choix des auteurs étudiés : la pertinence de certains est difficile à saisir (ce qu'Hans-Jörg Rheinberger reconnaît d'ailleurs à plusieurs reprises) et la vision d'ensemble est très continentale (le «programme 
fort » de David Bloor n'est par exemple évoqué que dans la toute dernière page du dernier chapitre, malgré l'importance de la tradition de recherche qu'il a suscitée ${ }^{2}$ ). Cet ouvrage a donc laissé le recenseur sur sa faim; mais des lecteurs plus compétents que lui en histoire des sciences et en épistémologie y trouveront certainement davantage de satisfaction.

\footnotetext{
${ }^{2}$ Il est notamment connu pour en avoir mis en avant les principes de symétrie et de réflexivité, selon lesquels les théories scientifiques ne doivent pas être analysées différemment selon qu'elles ont été reconnues vraies ou fausses, et d'une manière qui puisse rendre compte de leur analyse ellemême. On en trouve une présentation très claire dans l'ouvrage recensé ici : http://lectures.revues.org/251
} 